\title{
A violência no estado do Paraná: uma análise espacial das taxas de homicídios e de fatores socioeconômicos
}

\author{
Violence in the state of Paraná: a spatial analysis of de homicide rates and \\ socioeconomic factors
}

Wander Plassa ', José Luiz Parré "I

\begin{abstract}
Resumo
Observando as altas taxas de homicídios no estado do Paraná em meados de 2010, o objetivo deste trabalho foi, considerando a teoria da desorganização, analisar a relação entre fatores socioeconômicos e as taxas de homicídio. Para tal, diante da constatação de que a criminalidade tem uma geografia, além da análise fatorial, nos procedimentos metodológicos também foram utilizados a Análise Exploratória de Dados Espaciais (AEDE) e modelos econométricos espaciais. O valor do I de Moran foi positivo e significativo, indicando que há uma dependência espacial positiva na taxa de homicídio do Paraná, em que Clusters do tipo alto-alto foram presenciadas na região metropolitana de Curitiba e fronteira entre o Paraná e Paraguai. Nos resultados dos modelos econométricos espaciais, os fatores relacionados a desigualdade de renda (fator 2) e instabilidade residencial (fator 3) foram significativos e positivos ao afetar as taxas de homicídios no estado, corroborando a teoria da desorganização social. Ainda, através do modelo de dependência espacial na variável dependente, as taxas de homicídios de um município foram impactadas positivamente pela criminalidade observadas das suas vizinhas cidades.
\end{abstract}

Palavras-chave: Taxas de Homicídio. Análise exploratória de dados espaciais. Paraná.

\begin{abstract}
Considering the high homicide rates in the State of Paraná in mid-2010, the objective of this paper was, considering the social disorganization theory, to analyze the relationship between socioeconomic factors and the homicide rate. To this end, considering that crime has a geography, in the methodological procedures, in addition to the factorial analysis, the Exploratory Spatial Data Analysis (ESDA) and spatial econometric models were used. Moran's I value was positive and significant, indicating that there is a positive spatial dependence on the Paraná homicide rate, in which high-high Clusters were present in the metropolitan region of Curitiba and in the Paraná and Paraguay border. In the results of spatial econometric models, factors related to income inequality (factor 2) and residential instability (factor 3) were significant and positive in affecting homicide rates in the state, corroborating the social disorganization theory. Also, through the spatial dependence model in the dependent variable, the homicide rates of a municipality were positively impacted by the observed crime of its neighboring cities.
\end{abstract}

Keywords: Homicide rates. Exploratory spatial data analysis. Paraná state.

I Faculdade de Economia, Administração e Contabilidade de Ribeirão Preto da Universidade de São Paulo, SP, Brasil - wanderplasse@ outlook.com

II Universidade Estadual de Maringá, PR, Brasil - jparre@uem.br 


\section{Introdução}

O Atlas da violência (2018) mostra que as taxas de homicídio no Brasil vêm aumentando a cada ano. Altas taxas de homicídios, aparentemente um problema social, podem também ser consideradas um problema econômico. Segundo relatório "Custos Econômicos da Criminalidade no Brasil" (2018), os custos com a violência no País chegaram a cerca de $\mathrm{R} \$ 285$ bilhões no ano de 2015 , quase 4,5\% do PIB. Esse quadro, segundo Iaquinto (2014), impede que o Brasil alcance um maior progresso social e econômico. Santos e Kassouf (2008) apontam que uma das áreas afetadas pela violência é o mercado de trabalho, uma vez que incidem diretamente na redução da formação de capital humano, redução da atividade turística e a perda da atratividade de novos investimentos produtivos e/ou a expulsão dos existentes.

O "mapa da violência" (2016), pesquisa elaborada por Waiselfisz desde 1998, aponta que uma média de 115 pessoas foram vítimas de homicídio a cada dia em 2014 no Brasil, totalizando 42.291 pessoas assassinadas por armas de fogo. Segundo o relatório, considerando as mortes por arma de fogo, é a maior taxa de homicídio no período análise (21,2 a cada 100 mil habitantes) mesmo com as campanhas para desestimular a violência, como a do Desarmamento. No período de 2004-2014, foram computados cerca de 400 mil homicídios no país.

Ao comparar as taxas de homicídios por 100 mil habitantes com outros 200 países ${ }^{1}$, o Brasil aparece em oitavo lugar como mais violento para o período de 2000 e 2016, de acordo com dados da Organização Mundial de Saúde, com uma taxa de 29,5. Aqueles que apresentaram maiores taxas de homicídio em 2016, ano mais recente de análise, foram El Salvador $(82,8)$, Honduras $(56,5)$, Venezuela $(56,3)$, Jamaica $(47,0)$, Belize $(37,6)$, São Vicente e Granadinas $(36,5)$ e África do Sul $(34,0)$. Em números absolutos, porém, o país aparece com o maior número de vítimas no período, em que nesse último ano, mais de 60 mil pessoas foram vítimas de homicídio no Brasil.

No objeto de análise deste estudo tem-se o estado do Paraná, onde o número de vítimas em 2012 atingiu uma taxa maior que a média do país, 32,7 a cada 100 mil habitantes, totalizando 3.464 pessoas assassinadas só nesse ano (MAPA DA VIOLÊNCIA, 2014). O relatório ainda apresenta um cenário expressivamente negativo para o estado em 2010. A capital, Curitiba, apresentou a segunda maior taxa de homicídio por 100 mil habitantes entre as capitais brasileiras, 55,9. Destacou-se também duas cidades paranaenses entre as mais violentas do país, Campina Grande do Sul (região metropolitana de Curitiba) e Guaíra (Fronteira do estado com o Paraguai), com 130 e 112,8 vítimas a cada 100 mil habitantes, respectivamente.

Desta forma, objetiva-se analisar a distribuição espacial das taxas de homicídios no estado do Paraná para o ano de 2010, verificando a existência de Clusters, bem como examinar o nível de associação entre variáveis socioeconômicas observadas na literatura. Para atingir esse objetivo, são utilizados a análise fatorial, Análise Exploratória de Dados Espaciais (AEDE) e os modelos econométricos espaciais, como procedimentos metodológicos. A inovação desse artigo está em estudar o comportamento espacial de um estado pouco considerado em temas relacionados à violência. Concomitantemente, busca-se empregar índices socioeconômicos nesta análise, fato pouco discutido na literatura nacional.

O artigo está organizado em 5 seções, além desta introdução que procura apontar a situação brasileira e paranaense quanto as altas taxas de homicídios. A segunda seção focaliza na literatura internacional e nacional que trataram do crime, dos homicídios e da questão espacial na qual os crimes estão inseridos. Na seção seguinte é relatado a metodologia utilizada na pesquisa. A parte 4 apresenta os resultados da pesquisa e, por fim, a conclusão.

\section{Revisão de literatura}

\section{Fundamentação teórica}

Estudos sobre a importância de fatores econômicos na determinação da criminalidade começaram a ser desenvolvidos, a princípio, por Fleisher (1963), em seu artigo sobre os efeitos do desemprego na delinquência juvenil. O autor observou se as políticas públicas se destinavam a facilitar a entrada de jovens na força de trabalho e se essas políticas eram capazes de reduzir a delinquência.

O clássico trabalho de Becker (1968), Crime and Punishment: an economic approach, foi um marco para o início dos estudos da criminalidade baseados em variáveis econômicas. Para o autor, o indivíduo se defronta com dois tipos de mercados: um de atividades legais e outro baseado em atividades criminais. O agente, comportando-se de maneira racional, irá cometer um crime se o ganho associado com esse mercado excede o custo de oportunidade do uso alternativo de recursos. Em resumo, o crime acontecerá em decorrência da interação entre três fatores: a)

1 Disponível em: https://dataunodc.un.org/crime/intentional-homicide-victims. Acesso em 19 jun. 2019. 
custo moral da atividade criminal; b) a probabilidade de punição; e c) os incentivos econômicos do envolvimento em atividades criminais.

O trabalho de Becker deu suporte a outros trabalhos posteriormente desenvolvidos nessa área. Ehrlich (1973), foi o primeiro autor a desenvolver um modelo preciso para entender a participação em atividades criminosas. $\mathrm{O}$ indivíduo alocaria seu tempo em três setores: lazer, trabalho em atividades legais ou em atividades criminosas. Os agentes perceberiam a atividade criminal como uma atividade comum, contudo com um risco associado a essa atividade, o risco de prisão.

Realizando uma análise mais pontual e recente da teoria econômica para o crime no Brasil, Uchôa e Menezes (2012) encontraram duas vertentes para explicar o crime: a teoria da tensão (strain) de Merton (1938) e a teoria da desorganização social de Shaw e Mckay (1942). Em cada linha, uma instituição diferente seria responsável pela implementação de políticas para o combate ao crime. A desorganização social, segundo os autores, vê o problema da violência sendo causado, na maior parte, por fatores sociais e por isso, formalmente chamada de "doença" social. A pobreza, a desigualdade de renda e a extrema pobreza seriam responsáveis pela insegurança e instabilidade social. As ferramentas para combater o problema da violência deveriam ser oriundas de políticas públicas.

A solução para a redução da violência não estaria apenas em mudanças sociais, mas também em reformas individuais focando a reeducação e ressocialização criminal para a vida em sociedade. Em adição, políticas para criar trabalho, combate à fome e a pobreza seriam necessários para opor-se aos seus efeitos imediatos.

Em contrapartida, a teoria da tensão entende que o problema da violência é causado pela ineficiência das políticas de segurança pública. Os crimes ocorrem quando encontram condições ideais para acontecer, principalmente, em um ambiente de desrespeito às normas sociais. A solução para a criminalidade seria baseada em política de segurança pública mais eficaz, que aumente o custo da atividade criminal, desta forma, desencorajando-a. A questão fundamental nesta teoria não é somente a repreensão, mas o aumento do custo do crime, que faz dele menos vantajoso.

Deve-se observar também que a criminalidade tem uma geografia (ANDRESEN, 2006). O autor realizou uma pesquisa para analisar os aspectos espaciais da atividade criminal na cidade de Vancouver. O ponto de partida, segundo o autor, para pesquisas que estudam a criminalidade seria que o comportamento humano é situado em um lugar. Portando, o lugar onde o crime ocorreu deve ser uma das dimensões da investigação do crime.

\section{Fundamentação empírica}

Como verificado, há diferentes vertentes para se analisar os determinantes do crime. Utilizando a teoria da desorganização social e análise espacial para Chicago, Estados Unidos, por exemplo, Wang e Arnold (2008) encontraram que a desvantagem concentrada, uma medida de desigualdade entre áreas contíguas, é um forte preditor de taxas de homicídio. Di Tella e Schargrodsky (2004), analisando um ataque ao principal centro judaico na cidade de Buenos Aires, Argentina, em julho de 1994, encontraram efeito negativo da presença mais intensa da polícia no número de roubo de carros, nos bairros analisados. Bairros vizinhos, no entanto, não experimentam menos roubos de carros em relação ao resto da vizinhança.

No Brasil alguns autores analisaram o comportamento entre determinantes do crime e variáveis socioeconômicas. Andrade e Lisboa (2000) estudaram a faixa etária de 15 a 19 anos nos estados de Minas Gerais, São Paulo e Rio de Janeiro para os anos de 1981 a 1997. Os autores verificaram que o aumento do salário real e uma queda na desigualdade de renda diminuíram a taxa de homicídio. Um ponto que surpreendeu os autores foi que a queda do desemprego aumentaria a taxa de homicídio.

Araújo e Faijnzylber (2001) em um trabalho considerando os estados da federação, buscaram explorar o que causaria a criminalidade violenta no Brasil para os anos de 1981 a 1996. Os autores notaram, abordando determinantes econômicos, sociais e demográficos das taxas brutas de homicídio, diferentes resultados no modelo econômico, dependendo da idade do indivíduo, no qual desigualdade, níveis de renda e desemprego foram os fatores mais relevantes para explicar a violência.

Considerando o fator espacial, Barros et al. (2019) analisaram a distribuição espacial dos homicídios de todos os 5.595 municípios brasileiros. Os autores criaram um índice de desenvolvimento econômico, em que aqueles municípios com maior índice de desenvolvimento apresentavam menor incidência de criminalidade. Também é possível destacar trabalhos analisando regiões brasileiras, como Menezes et al. (2013), Plassa, Paschoalino dos Santos (2019), Barbosa e Alves (2010), Oliveira (2008) e Sass, Porsse e da Silva (2016). Menezes et al. (2013) realizaram um trabalho para analisar a correlação espacial entre homicídio e desigualdade social para a cidade do Recife. Os autores constataram que áreas com baixas taxas de homicídios são rodeadas por áreas com altas taxas de homicídios, fenômeno que os autores chamaram de "ilhas de segurança".

Plassa, Paschoalino dos Santos (2019) buscam entender os determinantes das altas taxas de homicídio na região nordeste brasileira. Os autores encontraram que dentre as variáveis socioeconômicas que impactam positivamente o homicídio na região, a desigualdade de renda foi uma das mais importantes para explicar altas taxas de homicídio 
em uma localidade. Utilizando modelos econométricos espaciais, os autores também encontraram que os efeitos indiretos, que decorrem do impacto sobre a variável dependente de uma unidade ocasionado por mudanças nas variáveis vizinhas à essa unidade, inflam ainda mais o impacto da desigualdade de renda na região.

A região sudeste, representada pelo estado de Minas Gerais, também foi objeto de análise espacial da criminalidade. Barbosa e Alves (2010) fizeram uso de técnicas espaciais e encontraram que crimes contra a vida estavam localizados em regiões mais pobres do estado. Enquanto àqueles relacionados a propriedade estavam mais presentes em cidades mais ricas. Oliveira (2008) buscou fazer uma análise espacial da criminalidade no Estado do Rio Grande do Sul e encontrou papel relevante da análise espacial e da desigualdade de renda em impactar a criminalidade nas cidades do estado. Por fim, Sass, Porsse e da Silva (2016) também analisando um estado da região sul do Brasil, o estado do Paraná, verificaram que as taxas de homicídios estavam mais relacionadas a taxa de pobreza e grau de urbanização.

Este trabalho procura complementar a literatura que analisa criminalidade no Brasil, ao analisar como o padrão espacial da taxa de homicídio se comporta no estado do Paraná, bem como dos fatores socioeconômicos. Para tanto, o trabalho fez uso da análise espacial com o suporte da teoria da desorganização social, junto com o emprego de índices socioeconômicos, conforme realizado por Wang e Arnold (2008) para um caso internacional.

\section{Referencial metodológico}

\section{Base de dados}

A variável dependente deste trabalho, taxa bruta de homicídio por 100 mil habitantes, foi coletada para os 399 municípios do Paraná. Para evitar volatilidade, foi calculada a mediana dos homicídios dos anos de 2008 a 2012. A base dos dados de óbitos foi retirada do Sistema de Mortalidade (SIM), do DataSUS, que é gerado pelo departamento de Situação de Saúde, para os anos de 2008 a 2012. Na base SIM foram utilizados os dados na $10^{a}$ versão da classificação Internacional de Doenças (CID-10), capítulo XX (Causas externas - agressões (Homicídios Dolosos)), que corresponde aos códigos X85 a Y09. Posteriormente, foi realizado o cálculo da taxa de homicídio por 100 mil habitantes utilizando estimativas intercensitárias disponibilizada pelo DataSUS.

De acordo com a teoria da desorganização, as variáveis explicativas são selecionadas para representar características e estruturais e socioeconômicas que afetariam as taxas de crime. Neste trabalho as variáveis estruturais e socioeconômicas estão apresentadas na tabela 1 e têm como principal fonte primária o Censo (1991, 2000 e 2010) realizada pelo Instituto Brasileiro de Geografia e Estatística (IBGE). Ademais, é apresentado o sumário da média e do desvio padrão de cada variável usada nesta pesquisa. Como o número de variáveis é grande e há considerável colinearidade entre elas, a análise fatorial foi realizada para consolidá-las em poucos fatores, formando, para o presente caso, três fatores: 1) Índices Municipais; 2) Desvantagens Concentradas; e c) Instabilidade Residencial.

Tabela 1 - Descrição das variáveis utilizadas

\begin{tabular}{|c|c|c|c|c|}
\hline Fatores & Variável & Descrição & Média & $\begin{array}{c}\text { Desvio } \\
\text { Pardrão }\end{array}$ \\
\hline \multirow{6}{*}{$\begin{array}{l}\text { Índices } \\
\text { Municipais }\end{array}$} & Densidade & $\begin{array}{c}\% \text { da população em domicílios com } \\
\text { densidade }>2\end{array}$ & 17,00 & 5,79 \\
\hline & Fecund & Taxa de fecundidade total em 1991 & 3,03 & 0,55 \\
\hline & Fundcompl & $\begin{array}{l}\% \text { de } 18 \text { anos ou mais com fundamental } \\
\text { completo }\end{array}$ & 3,03 & 2,03 \\
\hline & ExtremPobre & $\%$ de extremamente pobres & 3,40 & 3,45 \\
\hline & CrianExtremPobre & $\%$ de crianças extremamente pobres & 5,40 & 5,00 \\
\hline & RDPC & $\begin{array}{l}\text { RPD per capita em valores em R } \$ \text { de } 01 / \\
\text { agosto de } 2010\end{array}$ & 610,22 & 150,29 \\
\hline
\end{tabular}




\begin{tabular}{|l|cccc}
\hline \multirow{2}{*}{$\begin{array}{l}\text { Desvantagens } \\
\text { Concentradas }\end{array}$} & GINI & Índice de Gini & 0,47 & 0,06 \\
\cline { 2 - 5 } & Nbranco & Proporção de não brancos & 31,34 & 12,95 \\
\hline Instabilidade Residencial & Desocupado & Taxa de desocupação de 18 a 24 anos & 8,88 & 3,82 \\
\hline & MulherChef & $\begin{array}{c}\text { \% de crianças de 0 a 6 anos em } \\
\text { domicílios com responsáveis mulheres } \\
\text { em 2000 }\end{array}$ & 11,03 & 3,31 \\
\hline Fonte: Elaboração própria. & & & &
\end{tabular}

As variáveis Assistência (Assist), percentagem de extremamente pobres (ExtremPobre) e percentagem de criança extremamente pobres (CrianExtremPobre) foram extraídas do Censo de 2010. Para a variável assistência foi usado a proporção de famílias contempladas pelo Programa Bolsa Família (PBF) no ano de 2010. A variável "extremamente pobres" é a proporção dos indivíduos com renda domiciliar per capita igual ou inferior a $\mathrm{R} \$ 70,00$ mensais no ano de 2010. Crianças pobres é proporção dos indivíduos com até 14 anos de idade que têm renda domiciliar per capita igual ou inferior a $R \$ 70,00$ mensais no ano de 2010.

Dentre as variáveis demográficas, a densidade populacional (Densidade), incluída nesse trabalho, é baseada nos estudos de Glaeser e Sacerdote (1999). Espera-se que grandes cidades possuam altas taxas de densidades populacionais e desta forma altas taxas de homicídios. Para captar a densidade em cada município, optou-se por calcular a percentagem da população que vive em domicílio com mais de dois moradores por cômodos.

A variável Fecundidade (Fecund) foi extraída do Censo de 1991 e é baseada no trabalho de Hartung (2009). É esperado que, com uma alta taxa de fecundidade a 15 ou 20 atrás, tenha efeito hoje sobre as taxas de criminalidade. Outra variável incluída nesta pesquisa é a percentagem de pessoas de 18 anos ou mais com fundamental completo (Fundcompl), que fornece uma noção de variáveis educacionais em cada município. A renda per capita média (RDPC) é considerada uma medida de atividade da economia. Essa variável que foi retirada do censo de 2010, expressa em reais, é a razão do somatório da renda de todos os indivíduos e o número populacional total. Para verificar a distribuição de renda em cada município utilizou-se o índice de Gini (GINI) de 2010. O índice mede o grau de desigualdade existente na distribuição de indivíduos segundo a renda domiciliar per capita.

A proporção de não brancos (Nbranco) é composta por indivíduos que se autodeclararam pretos, pardos ou indígenas. A variável "desocupado" representa o percentual de pessoas acima de 10 anos que se encontra desempregada. E, por fim, a variável mulher chefe de família (MulherChef), obtida do censo de 2000, representa a taxa de crianças de 0 a 6 anos que residia em lares chefiados por mulheres, fornecendo uma noção de instabilidade familiar. Currie (1985) vê a relação de instabilidade familiar ao crime, nos distúrbios emocionais que a criança sofre durante separações familiares, e no aumento do risco da pobreza em casas chefiadas por mulheres.

\section{Análise fatorial}

A análise estatística multivariada é desmembrada em diversas técnicas, dentre elas está a Análise fatorial. Este estudo inclui a análise de componentes principais e análise dos fatores comuns. É adotada quando há um número grande de variáveis e correlacionadas entre si, com a finalidade de detectar um número menor de novas variáveis alternativas, não correlacionadas e que, de algum modo, condensem as informações principais das variáveis originais encontrando os fatores ou variáveis latentes (MINGOTI, 2005).

A composição de fatores ocorreria, segundo Farias, Figueiredo e Lima (2010), da seguinte maneira: as variáveis mais correlacionadas se ajustam dentro do mesmo fator e aquelas que compreende determinado fator são independentes das que constituem outro fator. Os componentes principais fazem com que o primeiro fator contenha o maior percentual de explicação da variância total das variáveis da amostragem; o segundo abriga o próximo maior percentual e assim por diante. Esse mecanismo permite a extração dos fatores de modo a maximizar a contribuição dos mesmos para a variância comum.

O método de análise fatorial pode ser expresso na forma matemática através de uma combinação linear entre as variáveis $\left(\mathrm{X}_{\mathrm{i}}\right)$ e $\mathrm{K}$ fatores comuns $(\mathrm{F})$.

$$
X_{i}=A_{i 1} F_{1}+A_{i 2} F_{2}+\cdots+A_{i k} F_{k}+U_{i}+E_{i}
$$

Onde Ai1 são as cargas fatorais usadas para combinar linearmente os fatores comuns; F1, F2, ..., Fk são os fatores 
comuns; A variância única Ui é a parte da variância total que não se relaciona com a variância das outras variáveis. Ei representa o erro de observação. No presente estudo, em que três fatores foram obtidos, tem-se que $\mathrm{k}=3$, conforme apresentado nos resultados deste trabalho. cargas fatoriais indicam o grau das relações entre as variáveis normalizadas Xi e os fatores. Quanto maior uma carga fatorial, mais ligada com o fator se encontra a variável. A comunalidade $\mathrm{hi}^{2}$ são índices concedidos às variáveis originais que expressam, em termos percentuais, o quanto da variabilidade de cada variável é explicada pela análise fatorial estimada. Quanto mais próximo da unidade esse índice melhor para o modelo.

A medida Eigenvalue ou raiz característica expressa a variância total do modelo explicada por cada fator. Ferreira Junior, Baptista e Lima (2004) sinalizam que na determinação do número de fatores necessários para representar o conjunto de dados, frequentemente é considerado apenas os fatores cuja raiz característica é maior que a unidade. O Eigenvalue dividido pelo número de variáveis $X_{i}$, determina a proporção da variância total explicada pelo fator.

Para verificar a adequação desse modelo à base de dados, aplicam-se dois testes: Kaiser-Meyer-Olkin (KMO), também conhecido como critério raiz latente, e Bartlett Test of Sphericity (BTS). O teste KMO se baseia na ideia de que existe uma estrutura de dependência bem definida entre as variáveis analisadas e que a mesma possa ser expressa pela matriz de correlações ou de covariância. A existência dessa estrutura implica que uma variável pode ser prevista pelas demais com certa margem de segurança. Assim, o modelo somente apresentará uma estrutura de dependência clara se a correlação parcial entre os pares de variância for baixa. O valor da estatística varia de zero a um, sendo que pequenos valores de KMO indicam que os dados não são adequados a análise.

O teste BTS testa a hipótese que a matriz de correlação é uma matriz identidade, ou seja, que não há correlação entre as variáveis. Cada variável se correlaciona perfeitamente com ela própria se $r=1$, mas não apresenta correlação com as outras variáveis se $r=0$. A significância para o teste não deve ultrapassar 0,05 .

\section{Análise espacial}

\section{Análise explanatória de dados espaciais e índice global e local de Moran}

A análise exploratória de dados espaciais (AEDE) segundo Anselin (1999), pode ser definido como uma coleção de técnicas para descrever e visualizar distribuição espacial, identificar localidades atípicas (Outliers espaciais), descobrir padrões de associação espacial (Clusters espaciais), sugerir diferentes regimes espaciais e outras formas de instabilidade espacial ou não estacionariedade espacial. O foco da AEDE é o conceito da autocorrelação espacial, que é o fenômeno onde, similaridades locacionais (observações em proximidade espacial) são combinadas por valores similares (correlação).

Um ponto importante a ser abordado antes de implementar as técnicas de análise explanatória dos dados espaciais é a definição de uma matriz de pesos espaciais $(\mathrm{W})$, que tem por objetivo capturar os efeitos de contiguidade e vizinhanças sobre os dados. Há na literatura algumas matrizes de pesos espaciais, no entanto, esse trabalho utilizará a matriz contiguidade binária do tipo rainha (Queen). Para verificar a dependência espacial, algumas estatísticas de testes são abordadas na literatura, como as estatísticas de $I$ de Moran, $C$ de Geary e o G de Getis-Ord. Apesar dessas abordagens preferiu-se a Estatística I de Moran por ser uma medida mais comumente usada (CLIFF e ORD, 1981).

O I de Moran representa um coeficiente de autocorrelação espacial usando a medida de autocovariância na forma de produto cruzado. De acordo com Santos e Raia Junior (2006), o índice de Moran é o mais utilizado quando se deseja um sumário da distribuição espacial de dados. Em termos formais e matriciais a estatística $I$ de Moran pode ser expressa como:

$$
I=\left(\frac{n}{S_{0}}\right)\left(\frac{z_{t}^{\prime} W z_{t}}{S_{0}}\right)
$$

em que,

$$
t=1, \cdots, n
$$

Onde o $n$ representa o número de áreas; $z$ denota os valores da variável de interesse padronizado; $W z$ representa os valores médios da variável de interesse padronizada nos vizinhos, definidos segundo uma matriz de ponderação espacial $W$. Os elementos $W_{i i}$ na diagonal são iguais a zero, enquanto os elementos $W_{i j}$ indicam a forma com a região $i$ está espacialmente conectada com a região $j$. O termo $s_{0}$ é um escalar igual à soma de todos os elementos de $W$.

O I de Moran tem um valor esperado de (-)[1/(n - 1)] conforme demonstrado por Cliff e Ord (1981), o que indica o valor obtido caso não houvesse padrão espacial nos dados. O valor calculado de $I$, segundo Almeida (2012), deve ser igual ao seu valor esperado, dentro dos limites da significância estatística.

Deve-se ter uma atenção especial com a interpretação do I de Moran uma vez que se trata de uma estatística de autocorrelação linear do tipo cruzado. A interpretação se divide em dois possíveis resultados: quando os valores de $I$ excedem o valor esperado e quando os valores do $I$ se encontram abaixo do valor esperado.

Um indicador local visa analisar localmente a associação espacial do lugar, baseada num indicador de concentra- 
ção espacial. É útil quando a estatística global não consegue captar eventuais bolsões localizados de concentração espacial. O Índice de Moran Local, proposto na literatura por Anselin (1999) faz uma decomposição do indicador global de autocorrelação na contribuição local de cada observação em quatro categorias (AA, BB, AB e BA). Alto-Alto (AA) significa que na região $i$ é alta em relação à média e seu vizinho $j$ também é alto. Baixo-Baixo (BB) indica que na região $i$ é baixa em relação à média e seu vizinho é baixo também e assim por diante.

Nos chamados indicadores LISA "Local Indicator of Spatial Association", a estatística deverá satisfazer dois critérios: a) capacidade, para cada observação, de indicar Clusters espaciais, significativos estatisticamente; b) propriedade de que o somatório dos indicadores locais, para todas as regiões, é proporcional ao indicador de autocorrelação espacial global correspondente.

A vantagem de um índice ser LISA é que quanto maior for o índice de Moran Global, esta não afetará as significâncias dos testes locais. Outra vantagem é poder captar uma associação espacial negativa, ou seja, os valores são dissimilares e os $I_{i}$ são negativos. Após computado os I de Moran local, deve-se verificar o seu nível de significância e plotá-los em um mapa. Com isso poderá observar as regiões de Clusters separadas, conforme as categorias citadas (AA, AB, BA e BB).

\section{Modelos econométricos}

Por fim, unindo a análise fatorial e espacial, para explicar a relação causal entre a taxas de homicídios no estado do Paraná, três modelos são estimados. O primeiro modelo, denominado de Mínimos Quadrados Ordinários (MQO), desconsidera qualquer relação espacial na estimação. O segundo modelo, conhecido como Defasagem Espacial (SAR), adiciona a defasagem da variável dependente na regressão. Por fim, o terceiro modelo, de Erro Espacial (SEM), considera a defasagem do termo de erro na regressão. Assim, o primeiro modelo, MQO, pode ser apresentado conforme a equação (3):

$$
y=\beta_{0}+A_{i 1} F_{1}+A_{i 2} F_{2}+A_{i 3} F_{3}+\varepsilon
$$

em que três fatores, $F_{1}, F_{2}$ e $F_{3}$ são considerados, conforme resultado obtido neste artigo. Ademais, $\varepsilon$ é o termo de erro com distribuição normal, média zero e variância constante. Contudo, o modelo MQO não será o melhor modelo se for confirmado a presença de autocorrelação espacial. Com esse intuito, os testes de Multiplicadores de Lagrange são realizados. O primeiro, ML (defasagem), investiga a defasagem espacial da variável dependente. Enquanto o segundo teste, ML (erro), a presença de defasagem espacial do termo de erro. A hipótese nula desses testes é não presença de defasagem espacial. Portanto, ao não rejeitar a hipótese nula de ambos os testes, o melhor modelo segue sendo o MQO. Ao se rejeitar apenas a hipótese nula do teste ML (defasagem), o formato da autocorrelação segue o modelo SAR, conforme a equação 4:

$$
y=\rho W y+\beta_{0}+A_{i 1} F_{1}+A_{i 2} F_{2}+A_{i 3} F_{3}+\varepsilon
$$

Em que $\rho$ é um escalar que mede o grau de autocorrelação espacial e W uma matriz espacial. No entanto, se ao contrário, apenas a hipótese nula do teste ML (erro) for rejeitada, o formato da autocorrelação segue o modelo SEM, conforme a equação 5:

$$
\begin{aligned}
& y=\beta_{0}+A_{i 1} F_{1}+A_{i 2} F_{2}+A_{i 3} F_{3}+u \\
& u=\lambda W_{\mu}+\varepsilon
\end{aligned}
$$

Em que $\lambda$ é o coeficiente do parâmetro do erro autorregressivo espacial que acompanha a defasagem $W \mu$. Caso ambas as hipóteses nulas forem rejeitadas, isto é, os testes forem significativos, deve-se recorrer a seus respectivos testes robustos. O mais significativo indica o modelo mais adequado.

\section{Resultados}

\section{Resultados da análise fatorial}

Foram considerados as variáveis cujo eigenvalue ou a raiz característica foram superiores a um, conforme discutido na metodologia deste estudo. As informações contidas nas 11 variáveis podem ser sumarizadas em três fatores, que possuem uma variância acumulada de $74,63 \%$. Esse valor é o montante que os três fatores conseguem explicar da variância total do modelo, como pode ser visto na tabela 2. 
Tabela 2 - Análise fatorial, raiz característica, variância explicada e acumulada

\begin{tabular}{cccc}
\hline Fatores & Raiz Característica & Variância Explicada (\%) & Variância Acumulada (\%) \\
1 & 5,11 & 46,44 & 46,44 \\
2 & 1,61 & 14,67 & 61,11 \\
3 & 1,48 & 13,52 & 74,63 \\
\hline \hline
\end{tabular}

Fonte: Resultados da pesquisa.

O fator 1 é responsável por 46,44,76\% da variância entre as variáveis. Esse fator é chamado de índices municipais pois ele captura, como pode ser visto na tabela 3, percentagem de participantes do PBF, densidade populacional, taxa de fecundidade, percentagem de pessoas de 18 ou mais anos com fundamental completo, extremamente pobres e crianças extremamente pobres e renda per capita. Nota-se, no entanto, que as variáveis Fundcompl e RDPC entram com sinais negativos, isto é, possuem uma relação inversa com o fator 1 . O fator 2 é causador de $14,67 \%$ da variância entre as variáveis e é denominado de desvantagens concentradas por englobar o índice de Gini e a taxa de não brancos na população. O fator 3 captura 13,52\% da variância, e pode ser rotulado de instabilidade residencial, por agrupar os desocupados e a porcentagem de crianças em famílias que possuem mulheres como chefe de família.

Tabela 3 - Análise fatorial - formação dos componentes

\begin{tabular}{lccc}
\hline \multirow{2}{*}{ Variáveis } & \multicolumn{3}{c}{ Componentes } \\
\cline { 2 - 4 } & Fator $\mathbf{1}$ & Fator 2 & Fator 3 \\
\hline Assist & 0,8676 & 0,1117 & $-0,0171$ \\
Densidade & 0,6991 & 0,2901 & 0,3061 \\
Fecund & 0,7717 & 0,1894 & 0,008 \\
Fundcompl & $-0,8521$ & 0,1109 & 0,2924 \\
ExtremPobre & 0,8172 & 0,4297 & $-0,1008$ \\
CrianExtremPobre & 0,7973 & 0,4387 & $-0,0621$ \\
RDPC & $-0,8621$ & 0,3435 & 0,0728 \\
GINI & 0,2163 & 0,8645 & 0,1164 \\
Nbranco & 0,3105 & $-0,5651$ & 0,4868 \\
Desocupado & $-0,0468$ & $-0,0063$ & 0,7907 \\
MulherChef & $-0,3972$ & 0,1206 & 0,7075 \\
\hline \hline
\end{tabular}

Nota: Método de extração: Análise do Componente principal; Método de rotação: Varimax com normalização de Kaiser.

Fonte: Resultados da pesquisa.

Tabela 4 - Testes de KMO e Bartlett

\begin{tabular}{lcc}
\hline \multicolumn{2}{c}{ Teste de KMO e Bartlett } \\
\cline { 1 - 2 } Medida Kaiser-Meyer-Olkin de adequação de amostragem. & 0,790 \\
\hline \multirow{2}{*}{$\begin{array}{l}\text { Teste de esfericidade de } \\
\text { Bartlett }\end{array}$} & Chi-quadrado aproximado & $5.572,986$ \\
& Sig. & 105,000 \\
\hline Fonte: Resultados da pesquisa.
\end{tabular}

Na tabela 4 pode-se verificar os testes de ajustamento do modelo. $O$ teste $K M O$, que representa a adequabilidade, indicou um valor de 0,790, o que pode ser considerado bom e a amostra é passível de ser analisada por técnicas de 
análise fatorial. O teste de Bartlett apresentou resultado significativo, rejeitando a hipótese nula de que a matriz de correlação é uma matriz identidade, isto é, de que não há correlação entre as variáveis.

\section{Taxas de homicídios no Paraná}

Criado os fatores e justificado o seu uso, o próximo passo é analisar o comportamento das taxas de homicídios no estado do Paraná. O mapa 1 apresenta os grupos de taxas medianas de homicídios nos municípios do Paraná no período 2008 a 2012. Os grupos foram divididos levando em consideração as o método de otimização de Jenks e as médias mundiais e brasileiras de homicídios².

Analisando cada agrupamento, verifica-se que no primeiro grupo estão as cidades que apresentaram uma taxa de homicídio mediana entre 0 e 8 homicídios por $100 \mathrm{mil} /$ habitantes, isto é, dentro dos padrões mundiais. Nesse grupo se verificou um total 160 municípios paranaenses (40,10\% do total), que podem ser considerados cidades com taxas "Muito Baixa" de homicídios. O grupo seguinte agrega aqueles municípios que tiveram uma taxa mediana de 9 até a 21 homicídios por 100 mil habitantes. O montante total nesse grupo foi de 34,08\% dos municípios paranaenses (136 municípios), que, neste trabalho, foi considerado taxa de homicídio "Baixa", pois estavam abaixo da média nacional desse período.

No terceiro grupo estão aqueles municípios que se enquadraram na faixa que vai de 22 até 38 homicídios por 100 mil habitantes. Nesse grupo estão 69 municípios que possuíram taxas de homicídios "Média". Desta forma o Estado do Paraná contava com 365 municípios na faixa que vai de Muito Baixa até a Média.

Os dois grupos seguintes possuem taxas de homicídios consideradas "Alta" e "Muito Alta" e somam 34 municípios (8,5\%) que estavam acimas das médias nacionais. Com taxas consideradas "Alta" estão os municípios que se encontram na faixa de 39 a 67 homicídios por 100 mil habitantes para o ano de 2010. Por último, as áreas mais escuras do mapa 1, encontram-se aqueles com taxa "Muito alta" de homicídios (68 - 123). O município com a pior taxa verificada no presente trabalho foi Campina Grande do Sul, região metropolitana de Curitiba, com uma taxa mediana considerável de 123,85 homicídios para cada 100 mil habitantes.

Pode-se verificar no mapa 1 que os municípios com taxa "Alta" de homicídios estão, na sua maior parte, próximos a regiões com taxas de homicídios consideradas "Muito alta", geralmente perto da capital do estado, Curitiba, e nas regiões de fronteira com o Paraguai. Esse padrão pode indicar dependência espacial entre esses municípios.

Mapa 1: Grupos de taxas de homicídios nos municípios do paraná, 2010

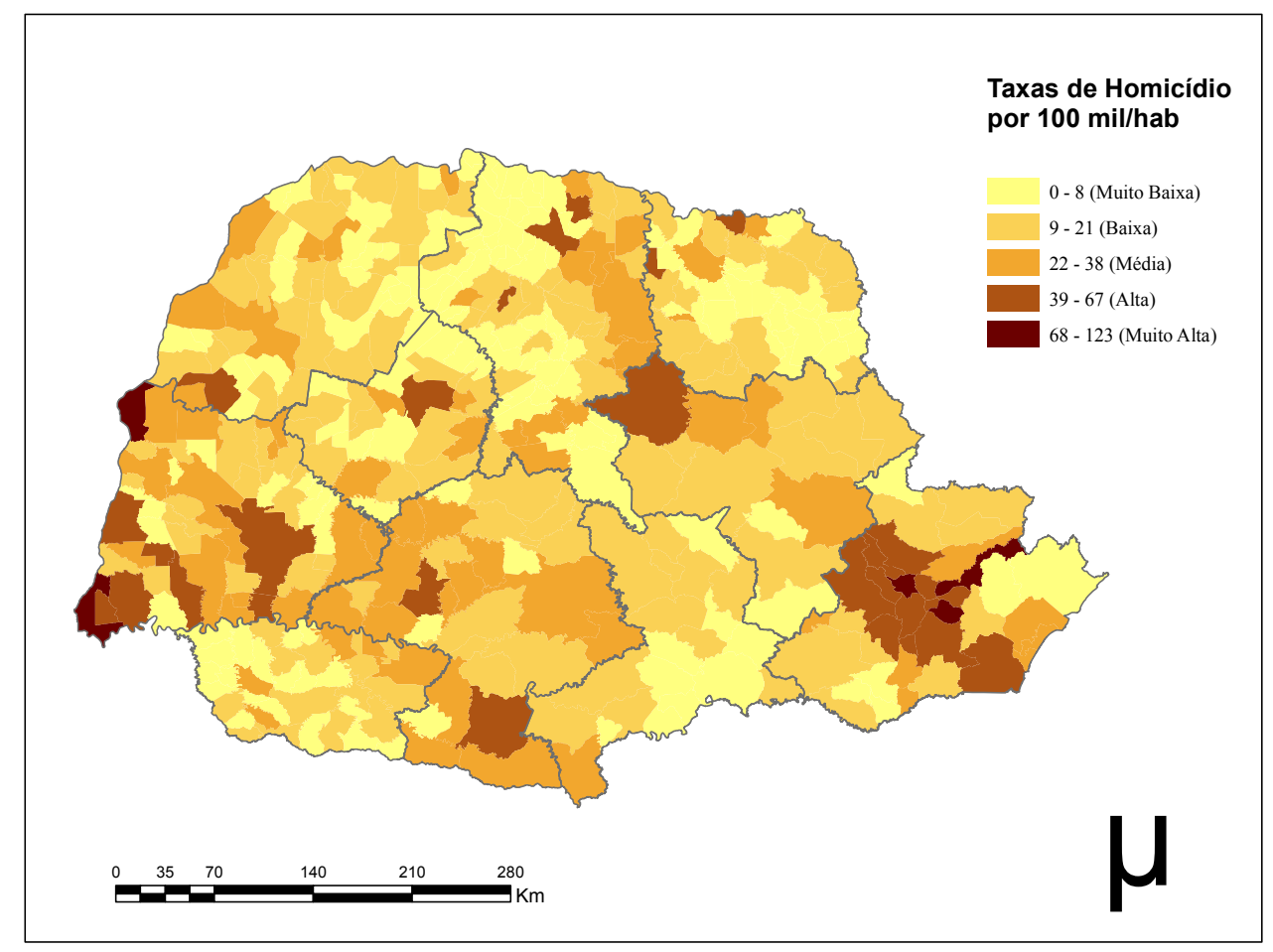

Fonte Resultados da pesquisa.:

2 A média mundial foi de 6,9 (estimações da Oficina de las Naciones Unidas contra la droga y el Delito) e a média brasileira 27,5 (Mapa da Violência) homicídios por 100 mil pessoas para 2010. Paralelo a isso, a Organizações das Nações Unidas (ONU) considera inaceitáveis taxas acima de 10 homicídios por grupo de 100 mil habitantes. 


\section{Efeitos espaciais sobre a taxa de homicídio}

\section{I de Moran global}

De acordo com os autores Griffith e Chavez (2004), o homicídio em uma determinada área pode se propagar para regiões vizinhas. Os autores argumentaram que vizinhanças que são geograficamente próximas a áreas violentas, independente do seu desenvolvimento social e estrutural, experimentam níveis altos de crimes e violência. Desta forma foi necessário a estimação de uma matriz de pesos como segue na tabela 5 .

Tabela 5 - Estatísticas Globais I de Moran para a variável correspondente à taxa de homicídio

\begin{tabular}{lccc}
\hline \multicolumn{1}{c}{ Matriz } & I & E[I] & P - Valor \\
Rainha & 0,3145 & $-0,0025$ & 0,001 \\
Bispo & 0,3146 & $-0,0025$ & 0,001 \\
K - 5 vizinhos & 0,3165 & $-0,0025$ & 0,001 \\
K - 7 vizinhos & 0,3185 & $-0,0025$ & 0,001 \\
K - 9 vizinhos & 0,3183 & $-0,0025$ & 0,001 \\
Nota: pseudossignificância empírica baseada em 999 permutações aleatórias. \\
Fonte: Resultados da pesquisa.
\end{tabular}

A matriz de pesos espaciais $W$ escolhida para a apresentação das análises espaciais e estimações dos modelos econométricos foi a matriz Binária do tipo Rainha, com valor de I de Moran de 0,3145 e estatisticamente significativa. Esse valor indica a autocorrelação global positiva pois excede seu valor esperado que é de $-0,0025$. Isso significa que, municípios que possuem altas taxas de homicídios estão próximos a regiões que também apresentam altas taxas de homicídios, isto é, há similaridade entre valores de uma determinada região e seus vizinhos. Porém nessa interpretação se perde a informação da força da autocorrelação, como constata Almeida (2012).

Na tabela 6 é apresentado o I de Moran Bivariado que, segundo Almeida (2012), tem como objetivo identificar se os valores de uma determinada variável observada em uma região apresentam relação com outras variáveis, também observadas, nas regiões vizinhas.

Tabela 6 - Coeficiente de I de Moran bivariado da taxa de homicídio no paraná e demais fatores

\begin{tabular}{lccc}
\hline \multicolumn{1}{c}{ Variáveis } & E $(I)$ & $I$ & Probabilidade \\
Fator 1 - Índices Municipais & $-0,0025$ & 0,0234 & 0,1260 \\
Fator 2 - Desvantagens Concentradas & $-0,0025$ & 0,1599 & 0,0010 \\
Fator 3 - Instabilidade Residencial & $-0,0025$ & 0,0827 & 0,0020 \\
\hline Nota: * pseudossignificância empírica baseada em 999 permutações aleatórias. &
\end{tabular}

Nota: * pseudossignificância empírica baseada em 999 permutaçôes aleatórias.

Fonte: Elaboração dos autores.

Há evidências de autocorrelação positiva entre as taxas de homicídio e o fator 1, que representa Índices Municipais. Isso significa que municípios que apresentaram altas (baixas) taxas de homicídios estão associados a municípios com altos (baixos) valores do fator 1. No entanto, através da análise de probabilidade esse fator não se mostrou significativo a $10 \%$. O fator 2 , que considera variáveis relacionadas principalmente a desigualdade de renda, também indicou essa característica com relação à taxa de homicídio. Porém nessa segunda análise a relação foi estatisticamente significativa. Mesmo resultado para o último fator analisado, Instabilidade Residencial, que se mostrou significantivo quando associado às taxas de homicídios.

I de Moran local

Segundo Almeida (2012) O I de Moran Local tem a capacidade de capturar padrões locais de associação linear. A maneira mais evidente de especificação da estatística se dá por meio de mapas. O Mapa 2 apresenta os Clusters univariados da taxa de homicídio. Nota-se que existem quatro Clusters classificados como Auto-Auto. O primeiro, refere-se à região Curitiba e sua região metropolitana: Almirante Tamandaré, Araucária, Bocaiúva do Sul, Campo Largo, Campo Magro, Colombo, Curitiba, Fazenda do Rio Grande, Itaperuçu, Mandirituba, Matinhos, Piraquara, Quatro Barras, Rio Branco do Sul, São José dos Pinhais e Tijucas do Sul, o que pode indicar um transbordamento da violência da capital do Paraná para as regiões vizinhas. 
Mapa 2: Clusters univariados da taxa de homicídio

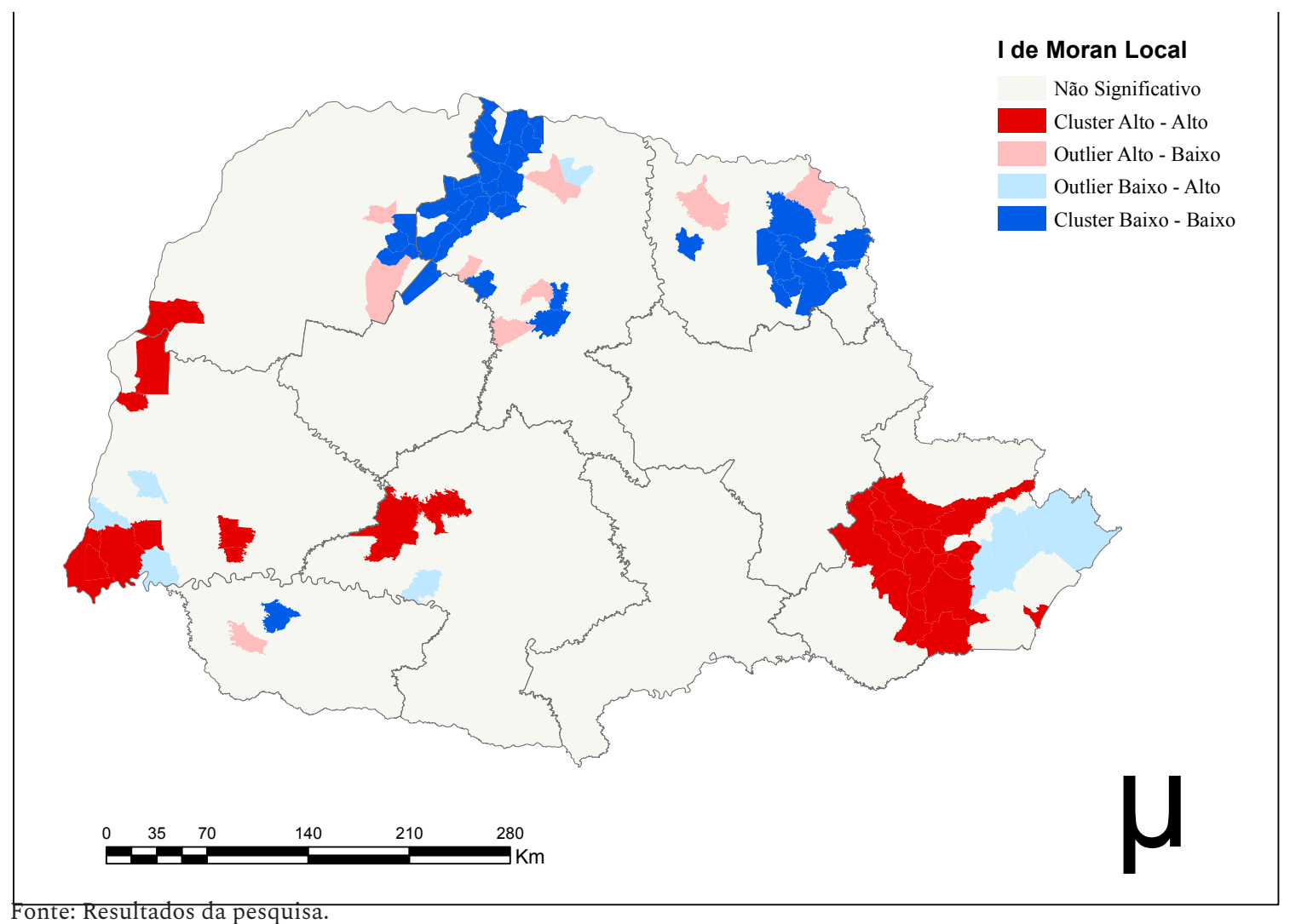

Outro agrupamento, localizado mais ao Centro do estado, é composto pelos municípios de Lindoeste, Marquinho, Nova Laranjeiras e Santa Lúcia, que estão no trecho que ligam a cidade de Foz do Iguaçu e a capital do estado. O último Cluster com essa classificação é representado pela fronteira do Paraná com o Paraguai, região de Foz do Iguaçu, formada pelas cidades de Altânia, Foz do Iguaçu, Medianeira, Mercedes, Santa Terezinha do Itaipu, São Miguel do Iguaçu e Terra Roxa.

Quanto ao agrupamento BB, verifica-se a existência de alguns Clusters desse tipo no norte Pioneiro e norte do estado. No norte do estado se constata um Cluster maior formado pelos municípios de Ângulo, Atalaia, Borrazópolis, Cafeara, Colorado, Flórida, Iguaraçu, Itaguaí, Itambé, Japur, Lobato, Lupionópolis, Mandaguaçu, Nova Senhora das Graças, Nova Esperança, Novo Itacolomi, Ourizona, Presidente Castelo Branco, Santa Fé, Santo Inácio, São Carlos do Ivaí, São Jorge do Ivaí, São Tomé e Terra Boa. Já no norte pioneiro, os municípios Carlópolis, Conselheiro Mairinck, Guapirama, Jaboti, Japirá, Jundiaí do Sul, Pinhalão, Quatigá, Santo Antônio da Platina, São Sebastião da Amoreira, Siqueira Campos e Tomazina, formam o outro Cluster BB no estado.

No Mapa 3 são apresentados os Clusters Bivariados formados pela associação entre as taxas de homicídio e os três fatores abordados nesta pesquisa. Essa estatística apresenta o grau de associação linear (positiva ou negativa) entre o valor de uma variável em uma determinada região e a média de uma outra variável nas localizações vizinhas.

Ao analisar a associação entre a taxa de homicídios e o fator 1, observa-se a presença de três clusters do tipo Alto-Alto, localizados na região central e centro sul do estado. O que indica que municípios com altas taxas de homicídios são rodeados por municípios com altos valores do fator 1 (índices municipais). Nas regiões mais violentas do estado, região metropolitana de Curitiba e a fronteira do estado com o Paraguai, observa-se a existência de Clusters $\mathrm{AB}$, em que há a presença de municípios com altas taxas de homicídios, rodeados por municípios com baixos valores de índices municipais.

Ao analisar a associação entre as taxas de homicídios e o fator 2, relacionado a desigualdade de renda, pode observar três grandes Clusters do tipo AA no estado. Um deles está localizado na região centro-sul, o segundo nas regiões de fronteiras do estado e o terceiro nas proximidades da região metropolitana da capital. Por último, é apresentado a associação entre taxas de homicídios e o fator 3 , ligado a instabilidades na residência. Clusters isolados do tipo Alto-Alto são percebidos principalmente na região metropolitana de Curitiba. 
Mapa 3: Clusters bivariados entre da taxa de homicídio e fatores socioeconômicos

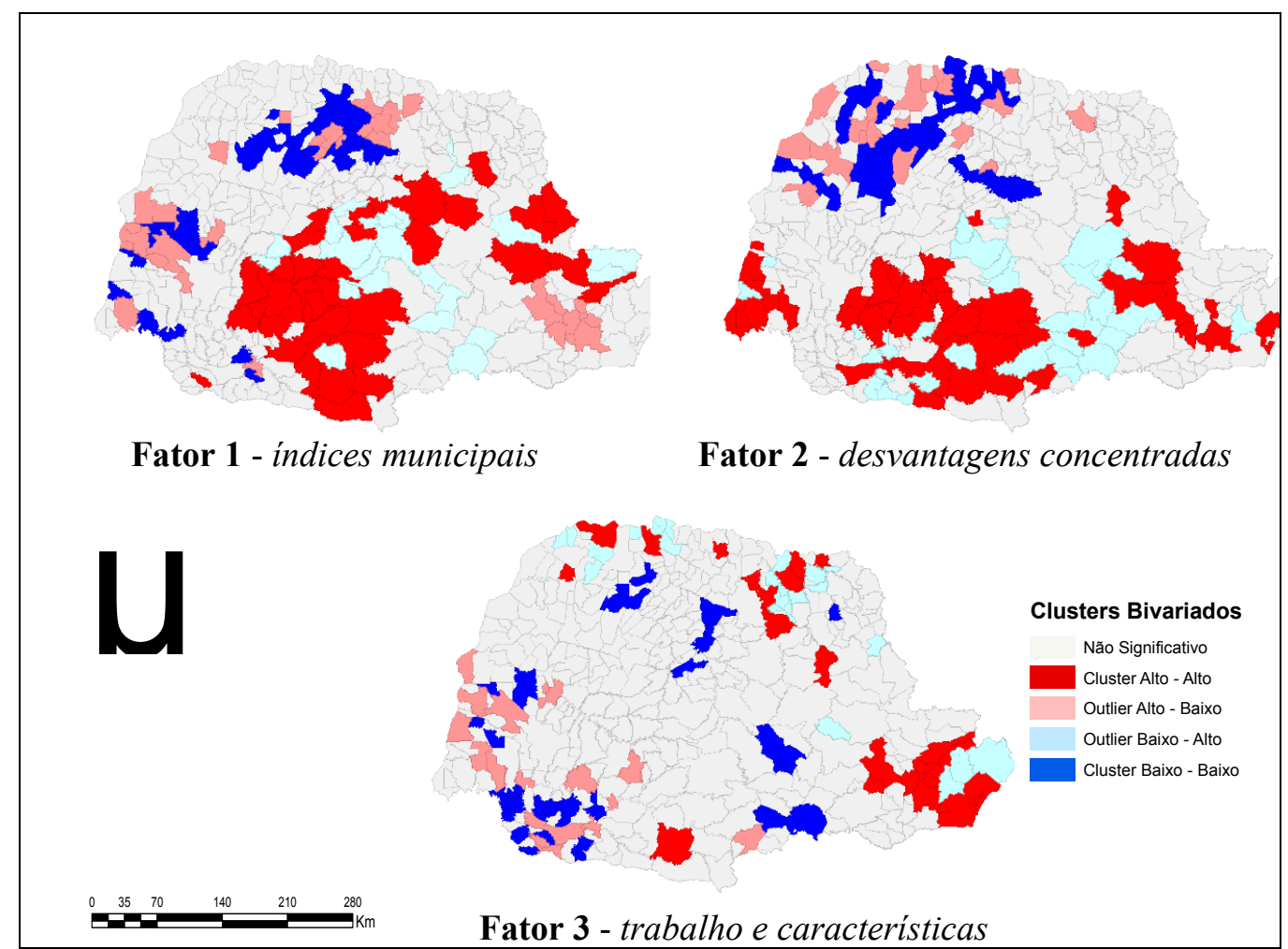

Fonte: Resultados da pesquisa.

\section{Modelos Espaciais Econométricos}

Observado a associação espacial entre as taxas de homicídios no estado e os fatores socioeconômicos, busca-se encontrar uma possível relação causal entre os fatores criados e as taxas de homicídios no estado. O primeiro passo foi identificar a existência de autocorrelação espacial e seu formato através do teste de $I$ de Moran com os resíduos do MQO e os testes robustos do Multiplicador de Lagrange, apresentados na Tabela 6.

Tabela 6: Diagnóstico para autocorrelação espacial

\begin{tabular}{lccccc}
\hline \multicolumn{1}{c}{ Diagnóstico } & Rainha & Torre & K-5 & K-7 & K-9 \\
& $8,653^{* * *}$ & $8,610^{* * *}$ & $8,478^{* * *}$ & $10,197^{* * *}$ & $11,411^{* * *}$ \\
I de Moran dos erros & $(0,000)$ & $(0,000)$ & $(0,000)$ & $(0,000)$ & $(0,000)$ \\
ML (Defasagem) & $81,779^{* * *}$ & $80,926^{* * *}$ & $81,246^{* * *}$ & $111,658^{* * *}$ & $137,367^{* * *}$ \\
& $(0,000)$ & $(0,000)$ & $(0,000)$ & $(0,000)$ & $(0,000)$ \\
ML (Defasagem) robusto & $12,316^{* * *}$ & $12,142^{* * *}$ & $16,220^{* * *}$ & $18,470^{* * *}$ & $22,963^{* * *}$ \\
& $(0,000)$ & $(0,000)$ & $(0,000)$ & $(0,000)$ & $(0,000)$ \\
ML (Erro) & $69,756^{* * *}$ & $69,082^{* * *}$ & $66,128^{* * *}$ & $93,599^{* * *}$ & $114,718^{* * *}$ \\
& $(0,000)$ & $(0,000)$ & $(0,000)$ & $(0,000)$ & $(0,000)$ \\
ML (Erro) robusto & 0,292 & 0,297 & 1,102 & 0,412 & 0,314 \\
\hline Nota: Em parênteses estão os valores das probabilidades. & $(0,585)$ & $(0,293)$ & $(0,520)$ & $(0,574)$ \\
\hline
\end{tabular}

Fonte: Elaboração dos autores.

Em todas as convenções os testes de $I$ de Moran foram significativos, o que aponta para a existência de uma 
autocorrelação nos erros. Os testes ML (Defasagem) e ML (Erro) também apresentaram significância a 1\%. Por fim, ao olhar para os testes robustos (ML (Defasagem) robusto e ML (Erro) robusto), notou-se que apenas o teste ML na defasagem foi significativo. Portanto, o modelo mais adequado no caso analisado é aquele que considera uma dependência espacial na variável dependente, isto é, o modelo SAR.

Não obstante, na Tabela 7 os três modelos são apresentados, em que a variável dependente, taxa de homicídios por $100 \mathrm{mil} /$ habitantes, foi calculado através da mediana dos anos de 2008 a 2012. O primeiro fator, que agrega variáveis relacionadas principalmente a pobreza, não apresentou significância estatística nos três modelos analisados. Esse resultado indica que a extrema pobreza e variáveis relacionadas a ela não aparenta explicar as altas taxas de homicídios em cidades do estado do Paraná. A teoria da desorganização social, de Shaw e Mckay (1942), defende que essas variáveis, por causar instabilidade social, podem causar o aumento da violência, o que não é observado neste estudo.

Porém, para a teoria da desorganização, variáveis relacionadas a desigualdade de renda também podem explicar altas taxas de violência em algumas localidades. Essas variáveis, que são consideradas no segundo fator, são denominadas de Desvantagens Concentradas. Esse fator foi significativo e positivo nos três modelos analisados. Isto é, quando maiores as desvantagens observadas em uma localidade, maiores serão as taxas de homicídios. Corroborando, pelo menos em parte, com a teoria da desorganização social e estudos nacionais que enfatizam a importância da desigualdade de renda na explicação das altas taxas de criminalidade (ANDRADE; LISBOA, 2000; OLIVEIRA, 2008; PLASSA; PASCHOALINO; DOS SANTOS, 2019).

Tabela 7: Resultados das regressões utilizando como método de estimação MQO, SAR e SEM

\begin{tabular}{lccc}
\hline \multicolumn{1}{c}{ Variáveis } & MQO & SAR & SEM \\
\hline \multirow{2}{*}{ Constante } & 6,421 & $-4,183$ & $-2,872$ \\
& $(0,181)$ & $(0,333)$ & $(0,552)$ \\
Fator 1 - Índices Municipais & 0,666 & 0,842 & 1,555 \\
& $(0,580)$ & $(0,434)$ & $(0,212)$ \\
Fator 2 - Desvantagens & $4,239^{* * *}$ & $3,183^{* * *}$ & $2,123^{* * *}$ \\
Concentradas & $(0,000)$ & $(0,000)$ & $(0,010)$ \\
& $3,516^{* * *}$ & $2,372 * * *$ & $2,110^{* *}$ \\
Fator 3 - Instabilidade Residencial & $(0,000)$ & $(0,009)$ & $(0,038)$ \\
& - & $0,478^{* * *}$ & - \\
$\rho$ & - & $(0,000)$ & - \\
& - & - & $0,540^{* * *}$ \\
\hline
\end{tabular}

\begin{tabular}{lccc}
\hline & \multicolumn{2}{c}{ Testes } & \\
\hline Log Likehood & $-1661,41$ & $-1628,95$ & $-1627,31$ \\
Critério de Akaike & 3332,83 & 3269,90 & 3264,63 \\
Critério de Schwarz & 3352,77 & 3293,84 & 3284,57 \\
$\mathrm{R}^{2} /$ Pseudo R & 0,143 & 0,306 & 0,322 \\
\hline Núm. Observações & 399 & 399 & 399
\end{tabular}

Núm. Observações

399

399

Nota: Todas as variáveis estão em logaritmos. Em parênteses estão os valores das probabilidades. ${ }^{\star \star \star \star} \mathrm{p} \leq 0,01,{ }^{{ }^{*}} \leq 0,05,{ }^{*} \leq 0,10$. A taxa de urba nização de cada município foi utilizada como controle nos três modelos.

Fonte: Elaboração dos autores.

O último fator analisado, chamado de Instabilidade Residencial, considera variáveis que podem refletir na instabilidade nas residências, como por exemplo, a perda de um emprego ou mesmo a ocorrência de divórcios. Esse fator também se mostrou relevante para explicar as taxas de homicídios no Paraná. Conforme observado na Figura 3, quando analisado esse fator, clusters do tipo Alto-Alto foram formados principalmente na região metropolitana de Curitiba, região que possuí as cidades mais violentas do estado. Os resultados da Tabela 7 confirmam que as insta- 
bilidades observadas na residência podem estar implicando em maiores taxas de homicídios no estado. Conforme discutido pela teoria da desorganização, o incentivo à criação de empregos na região, pode ser um dos fatores que contribuem para a redução da violência no estado.

Por fim, o valor estimado de $\nabla$, que mede o grau de autocorrelação espacial, também foi estatisticamente significativo e positivo. Esse resultado implica que parte da violência observada em um município pode ser explicada pelas altas taxas de violências reportadas nos municípios vizinhos. O que demonstra a importância do combate a violência não apenas à nível local, mas em conjunto com outros municípios que forma regiões metropolitanas.

\section{Considerações finais}

Utilizando as variáveis fundamentadas na teoria da desorganização social, o presente estudo buscou entender o impacto de variáveis socioeconômicas nas taxas de homicídios do estado do Paraná no período de 2010 . Para tanto, desenvolveu-se uma análise de distribuição espacial das taxas de homicídios em conjunto com a utilização de modelos de econometria espacial. Verificou-se, por exemplo, que as altas taxas de homicídios no estado estão concentradas nas regiões da capital e de fronteira com o Paraguai.

O teste de dependência global mostrou que existe uma dependência espacial positiva entre os municípios do estado do Paraná com relação as taxas de homicídio, em que municípios com altas (baixas) taxas de homicídios são circundados por municípios com altas (baixas) taxas de homicídios. Para explicar parte das altas taxas de homicídios em certas regiões do estado, fatores socioeconômicos, obtidos da análise fatorial, foram criados. Os fatores relacionados a desvantagens concentradas e instabilidade residencial tiveram um impacto positivo e significativo sobre as taxas de homicídios no estado, corroborando, em parte, as ideias defendidas pela teoria da desorganização social. Também, identificou-se, através do modelo SAR, que parte das taxas de homicídios em um local podem ser explicados pela violência observada em municípios vizinhos.

Os resultados encontrados neste trabalho ajudam a compreender, pelo menos em partes, as causas das altas taxas de homicídios em determinadas regiões do estado do Paraná ao considerar variáveis relacionadas a dados socioeconômicos. Variáveis ligadas ao desrespeito às normas sociais, consideradas na teoria da tensão, não foram objeto de estudo neste trabalho. Além disso, problemas relativos ao tráfico de drogas, tão comum em municípios de fronteira do estado com o Paraguai, também não foram considerados, diante da sua difícil mensuração. Configurando-se, esses, fatores limitadores deste estudo.

Mesmo dessas dificuldades, os resultados encontrados mostram que políticas públicas e de segurança seriam mais eficazes em reduzir a violência no estado do Paraná se contribuírem com a geração de emprego e com as reduções das desigualdades de renda, principalmente nos grandes centros. Ademais, o combate à violência deve ser uma política regional (e não apenas municipal) uma vez que a violência de cidades vizinhas tem impacto positivo sobre as taxas de homicídios de um determinado município.

\section{Referências}

ALMEIDA, E. Econometria espacial. Campinas-SP. Alínea, 2012.

ANDRADE, M. V.; Lisboa, M. B. Desesperança de vida: homicídio em Minas Gerais, Rio de Janeiro e São Paulo: 1981 a 1997. In: Anais do IX Seminário sobre a Economia Mineira [Proceedings of the 9th Seminar on the Economy of Minas Gerais]. Cedeplar, Universidade Federal de Minas Gerais, p. 775-808, 2000.

ANDRESEN, M. A. A spatial analysis of crime in Vancouver, British Columbia: A synthesis of social disorganization and routine activity theory. The Canadian Geographer/Le Géographe canadien, v. 50, n. 4, p. 487-502, 2006.

ANSELIN, L. Interactive techniques and exploratory spatial data analysis. Geographical Information Systems: Principles, Techniques, Management and Applications, eds., P. Longley, M. Goodchild, D. Maguire, and D. Rhind. Cambridge: Geoinformation Int, 1999.

ARAÚJO JUNIOR, A. D.; FAJNZYLBER, P. O que causa a criminalidade violenta no Brasil? Uma análise a partir do modelo econômico do crime: 1981 a 1996. Belo Horizonte: Universidade Federal de Minas Gerais, CEDEPLAR, 88p. Texto de Discussão, v. 162, 2001. 
BARBOSA BATELlA, W.; ALVES DiniZ, A. M. Análise espacial dos condicionantes da criminalidade violenta no estado de Minas Gerais. Sociedade \& Natureza, v. 22, n. 1, 2010.

BARROS, Pedro Henrique Batista de et al. Economic development and crime in brazil: a multivariate and spatial analysis. Revista Brasileira de Estudos Regionais e Urbanos, v. 13, n. 1, p. 1-22, 2019.

BECKER, G. S. Crime and punishment: An economic approach. In: The economic dimensions of crime. Palgrave Macmillan, London, 1968. p. 13-68.

CERQUEIRA, Daniel Coordenador et al. Atlas da violência 2018. Ipea e FBSP, 2018. Disponível em: http:// repositorio.ipea.gov.br/bitstream/11058/8398/1/Atlas\%20da\%20viol\%c3\%aancia_2018.pdf. Acesso em 08 ago. 2019.

CLIFF, A. D.; ORD, J. K. Spatial processes: models \& applications. London: Pion, 1981.

CURRIE, E. Confronting crime: an American challenge. New York: Pantheon Books, 1985.

DI TELLA, Rafael; SCHARGRODSKY, Ernesto. Do police reduce crime? Estimates using the allocation of police forces after a terrorist attack. American Economic Review, v. 94, n. 1, p. 115-133, 2004.

EHRLICH, I. Participation in illegitimate activities: A theoretical and empirical investigation. Journal of political Economy, v. 81, n. 3, p. 521-565, 1973.

FERREIRA JÚNIOR, S.; BAPTISTA, A. J. M. dos S.; LIMA, J. E. de. A modernização agropecuária nas microrregiões do Estado de Minas Gerais. Brazilian Journal of Rural Economy and Sociology (Revista de Economia e Sociologia Rural-RESR), v. 42, n. 1346-2016-105086, p. 73, 2004.

FARIAS, C. A.; FIGUEIREDO, A. M.; LIMA, J. E. d. Dependência espacial e análise de agrupamento de municípios para diferentes tipos de crime em Minas Gerais. REUNA, v. 13, n. 3, 2010.

FLEISHER, B. M. The effect of unemployment on juvenile delinquency. Journal of Political Economy, v. 71, n. 6, p. 543-555, 1963.

GLAESER, E. L.; SACERDOTE, B. Why is there more crime in cities?. Journal of political economy, v. 107, n. S6, p. S225-S258, 1999.

GRIFFITH, E.; CHAVEZ, J. M. Communities, street guns and homicide trajectories in Chicago, 1980-1995: Merging methods for examining homicide trends across space and time. Criminology, v. 42, p. 941, 2004.

HARTUNG, G. C. Ensaios em demografia e criminalidade. Tese de Doutorado. Escola de Pós-Graduação em Economia - EPGE. Fundação Getúlio Vargas, 2009.

IAQUINTO, K. Efeito dominó: o custo da violência. Revista Conjuntura Econômica, v. 68, n. 1, p. 20-27, 2014.

MENEZES, T. et al. Spatial correlation between homicide rates and inequality: evidence from urban neighborhoods. Economics Letters, v. 120, n. 1, p. 97-99, 2013.

MERTON, R. K. Social structure and anomie. American sociological review, v. 3, n. 5, p. 672-682, 1938.

MINGOTI, S. A. Análise de dados através de métodos de estatística multivariada: uma abordagem aplicada. Editora UFMG, 2005.

OLIVEIRA, C. A. de. Análise espacial da criminalidade no Rio Grande do Sul. 2008.

PLASSA, W; PASCHOALINO; P. A. T.; SANTOS, M. P. Determinantes Socioeconômicos das Taxas de Homicídios no Nordeste Brasileiro: Uma Análise Espacial. Planejamento e Políticas Públicas, n. 53, 2019. 
Secretaria Especial de Assuntos Estratégicos (SAE). Custos Econômicos da Criminalidade no Brasil. Presidência da República. Relatório de Conjuntura № 4, 2018. Disponível em: http://www.secretariageral.gov.br/estrutura/ secretaria_de_assuntos_estrategicos/publicacoes-e-analise/relatorio-de-conjuntura/custos_economicos_ criminalidade_brasil.pdf. Acesso em 17 jun. 2019.

SHAW, C. R.; MCKAY, H. D. Juvenile delinquency and urban areas. 1942.

SANTOS, M. J. d.; KASSOUF, A. L. Estudos econômicos das causas da criminalidade no Brasil: evidências e controvérsias. Revista EconomiA, v. 9, n. 2, p. 343-372, 2008.

SANTOS, L.; RAIA JUNIOR, A. Análise espacial de dados geográficos: a utilização da Exploratory Spatial Data Analysis-ESDA para identificação de áreas críticas de acidentes de trânsito no município de São Carlos (SP). Sociedade \& Natureza, v. 18, n. 35, 2006.

SASS, K. S.; PORSSE, A. A.; DA SILVA, E. R. H. Determinantes das taxas de crimes no Paraná: uma abordagem espacial. Revista Brasileira de Estudos Regionais e Urbanos, v. 10, n. 1, p. 44-63, 2016

UCHÔA, C. F. A.; TATIANA, M. The Crime Reduction's Determinants: A Spatial Panel Analysis For Brazilian States. SEA, 2012.

Waiselfiz, J. J. Mapa da violência 2016: homicídios por armas de fogo no Brasil. 2016. Disponível em: https://www. mapadaviolencia.org.br/pdf2016/Mapa2016_armas_web.pdf. Acesso em 08 ago. 2019.

Waiselfiz, J. J. Mapa da violência 2014: Os jovens do Brasil. 2014. Disponível em: https://www.mapadaviolencia. org.br/pdf2014/Mapa2014_JovensBrasil.pdf. Acesso em 08 ago. 2019.

WANG, F.; ARNOLD, M. T. Localized income inequality, concentrated disadvantage and homicide. Applied Geography, v. 28, n. 4, p. 259-270, 2008. 